\title{
Efectividad de las intervenciones de base comunitaria para la prevención de los actos suicidas. Una revisión sistemática ${ }^{1}$
}

\author{
Gloria Josefa Sánchez Marínez \\ Carlos Federico Barreto Cortés \\ Julio César Valderrama Lozano \\ Fabio Alexander Salazar Piñeros \\ Especialización en Epidemiología \\ Universidad Surcolombiana, Colombia \\ fabiosalazar@usco.edu.co
}

\section{Resumen}

El suicidio es una de las mayores preocupaciones a nivel mundial por la cantidad de vidas que se cobran anualmente por esta vía. Los esfuerzos de los organismos de salud se han encaminado a reducir este flagelo a través de diversas estrategias. Lamentablemente las estadísticas siguen en aumento. Las estrategias para atender el tema del suicidio son de diversa índole y van desde las netamente clínicas/individuales como el uso de medicamentos para tratar la depresión o técnicas de psicoterapia, hasta programas nacionales de restricción de medios. La presente revisión se propuso hacer un examen de la evidencia en torno a la efectividad de los programas de base comunitaria, teniend o como resultados primarios las tasas nacionales o regionales de suicidio o intento de suicidio (actos suicidas). Un total de 20 estudios fueron revisados, de los cuales en tan solo 9 midieron sus efectos sobre las tasas de incidencia de actos suicidas, mientras que los restantes midieron otras variables como conocimientos y actitudes frente al suicidio y la depresión. Los programas fueron probados a través de estudios cuasiexperimentales especialmente y queda en evidencia que falta aplicar métodos más rigurosos tanto en términos de control de sesgos como de evaluación de los efectos.

Palabras clave: efectividad, suicidio, intento de suicidio, prevención, base comunitaria

\section{Introducción}

La Organización Mundial de la Salud define el suicidio como cualquier acto intencional que tiene como fin terminar con la propia vida (1). Se constituye como una conducta antinatural dado que no es parte de la carga instintiva terminar con la vida por sí mismo. El suicidio conlleva una carga emocional alta para la mayoría de los sobrevivientes y, en muchos casos, las familias reprimen el evento en razón al estigma y al tabú que esta conducta representa en la sociedad; esto hace que el problema se complejice al involucrar elementos individuales, familiares, sociales y culturales, lo que obliga a que la atención sea igualmente multifactorial.
Cada año, más de 800 mil personas terminan con su vida, y las edades más frecuentes están entre los 15 a 29 años en países de alto ingreso y entre 30 y 49 años en países de mediano y bajo ingreso (1). Para el año 2015 fue la décima causa de muerte en Estados Unidos y la segunda en jóvenes de 15 a 24 años; además, 3,3 hombres se autoeliminaron por cada mujer (2). En los países de mediano y bajo ingreso de las américas, la tasa de incidencia fue de 6,1 por cada 100 mil habitantes en 2012 (1). Estas cifras hacen que el Suicidio sea una de las causas de muerte más frecuentes en personas en edad productiva $\mathrm{y}$, naturalmente, un problema de interés en salud pública.

1 Trabajo Grupal presentado como requisito para el curso Revisiones Sistemáticas. 
Cifras del Instituto Nacional de Medicina Legal y Ciencias Forenses indican que durante 2015 en Colombia se presentaron 2.068 casos de suicidio que corresponden a una incidencia de 5,22 por cada 100 mil habitantes (3); adicionalmente, el Sistema de Vigilancia Epidemiológica de Lesiones de Causa Externa (SIVELCE) de esta misma institución, reportó 18.618 suicidios ocurridos en Colombia durante el decenio 20062015, con una media de 1.862 eventos por año y el promedio de la tasa del período fue de 4,20 casos por cada 100.000 habitantes mayores de cinco años (3).

Los datos evidencian que en 2015 se alcanzó la cifra más elevada del decenio con un incremento absoluto frente a $2006 \mathrm{del} 18 \%$ y del $10 \%$ respecto de 2014 (3). Esto demuestra que, pese a todos los esfuerzos, la problemática del suicidio, en lugar de disminuir, incrementa dramáticamente.

Para el Departamento del Huila, la situación es similar o en algunos casos de mayor preocupación con respecto a los datos obtenidos de otros departamentos y hasta el mismo índice Nacional; se tiene que dieciséis departamentos ostentaron tasas (casos por cada 100 mil habitantes) superiores a la nacional, de los cuales los cinco con las tasas más elevadas fueron Arauca (11,68), Amazonas $(10,68)$, Caldas $(8,45)$, Huila $(7,75)$ y Quindío $(7,59)$. Es importante señalar que Arauca, Huila y Quindío, repitieron los cinco primeros puestos entre los departamentos con las mayores tasas de suicidio durante 2014 (3).

Estas cifras ponen de manifiesto el evento del suicidio como problema de salud pública de gran envergadura en el país y las graves afectaciones que está generando en los ambientes de la salud, la comunidad y las familias (4).

Diferentes perspectivas teóricas han intentado develar el origen y la dinámica del suicidio. No obstante, aún surgen diversas posturas sin llegar a un acuerdo. Así mismo, existen múltiples enfoques de intervención que pretenden dar manejo al problema pero es evidente, a la luz de las estadísticas mundiales, que este fenómeno crece o, en el mejor de los casos, se mantiene.
Es así como en mayo de 2013 la 66 $6^{\underline{a}}$ Asamblea Mundial de la Salud de la OMS adoptó el primer plan de acción sobre la salud mental, definiendo como una de sus metas para 2020, reducir en un $10 \%$ la tasa de los suicidios en los países (5) .

El interés en intervenciones para reducir el riesgo de suicidio va desde la perspectiva biomédica que se centra en el uso de sustancias como el Litio o los antidepresivos $(6,7)$, hasta las técnicas multimodales que aseguran que la combinación de diferentes tipos de estrategias resultan más efectivas $(8,9)$. Las técnicas psicosociales han tenido una acogida creciente, en especial aquellas provenientes del modelo cognoscitivo comportamental $(10,11)$.

Cada una de las perspectivas mencionadas asume que tiene como respaldo un corpus de conocimiento suficiente para satisfacer a sus respectivos seguidores y para no admitir debilidades que permitan el paso a otras alternativas. No obstante, es sabido que la línea más clínica tiene efectos individuales dignos de reconocer, pero el nivel poblacional no se favorece de manera equiparable. De otro lado, las estrategias multimodales que abordan el nivel comunitario no tienen un efecto directo sobre el individuo pero se beneficia el nivel de la salud pública. Ante la ambivalencia, conviene acudir a la frase clásica de Geoffrey Rose en 1984 "un gran número de personas en bajo riesgo pueden aportar más casos de una enfermedad que un pequeño número en alto riesgo"(12). Lo que Rose deja ver es la necesidad de invertir en estrategias poblacionales pues tendrán un mayor impacto, a la hora de abordar las problemáticas de salud pública, entre las cuales el suicidio juega un papel protagónico.

En la misma línea, la Asociación Internacional para la Prevención del Suicidio (IASP, por su nombre en inglés) reconoce como efectivas seis tipos de estrategias que desde el siglo pasado había propuesto la Organización Mundial de la Salud, a saber: tratamiento de las personas con trastornos mentales, control a la posesión de armas, detoxificación del gas doméstico, detoxificación de las emisiones de los vehículos, control de la disponibilidad de sustancias tóxicas y un manejo adecuado de los medios 
de comunicación (13). Si bien la primera de las estrategias es eminentemente clínica/ individual, las demás obedecen a acciones de base poblacional (incluyendo la formulación de políticas) para la prevención del suicidio. Esto guarda total coherencia con el modelo de Atención Primaria en Salud surgido como la gran propuesta para alcanzar la salud global desde Alma Ata en 1978, en el que invita, entre otros, a la implementación de programas de base comunitaria (14) pero que la falta de investigación rigurosa no ha permitido conocer de manera fiable su verdadero potencial en la reducción del Suicidio.

El término "base comunitaria" o "basado en comunidad" ha sido estudiado y definido por varios autores, quienes plantean que a la hora de la implementación de intervenciones en salud pública es preciso distinguir si Comunidad se refiere a un espacio geográfico, a un grupo de personas en un sentido más relacional, o a una entidad social con un compromiso político claro (15). Whitehead, por su parte, identifica hasta siete diferentes tipos de programas basados en comunidad (16). Cada una de estas perspectivas supone unos indicadores específicos para determinar su éxito o su fracaso. Los expertos reconocen que los elementos cruciales de una intervención basada en comunidad incluyen estrategias como movilización social, programas de autoayuda, uso de medios de comunicación, intervención en escenarios diversos como el lugar de trabajo, escuela, iglesia/culto, centros de salud, arreglo de ambientes como disposición de productos en los supermercados, menús en los restaurantes, políticas de fomento o restricción, etc. Del mismo modo, involucra a líderes comunitarios, redes sociales y cualquier tipo de organización comunitaria $(17,18)$.

La evidencia indica que muchos programas basados en la comunidad han tenido sólo un impacto modesto, con excepción de algunos programas de prevención del VIH. Los factores que llevarían a esta conclusión pesimista están relacionados con aspectos metodológicos como el bajo poder estadístico y la dificultad de implementar diseños rigurosos, las tendencias seculares, un reducido tamaño del efecto, debilidad en la aplicación de la intervención como insuficiente adaptación al contexto o poca penetración en la comunidad, y un sustento teórico deficiente debido a la complejidad de la naturaleza multinivel de muchas de las intervenciones (18). Frente a este último factor, es evidente que existe una brecha innegable entre la intervención y el resultado, toda vez que el diseño del programa no siempre coincide, en lo relativo a su marco conceptual, con los indicadores para medir su éxito. Se asume a priori la existencia de un papel mediador de ciertas variables (psicosociales, por ejemplo) sobre resultados comportamentales, sin observancia de los desarrollos teóricos al respecto (19). Para el caso presente y como se verá más adelante, varios de los programas se formularon para reducir el suicidio pero sus efectos se midieron en los cambios de actitudes hacia el suicidio, conocimientos sobre la depresión y el suicidio, solo por mencionar algunos ejemplos.

Pese al panorama negativo, el enfoque comunitario sigue siendo una apuesta global, pues se reconoce como una estrategia que reduce las inequidades en salud y de paso se instituye a la comunidad como parte de la solución en tanto sujeto activo (20). La Comisión sobre Determinantes Sociales de la Salud, creada desde la Organización Mundial de la Salud, planteó tres principios de acción fundamentados en el reconocimiento de la salud como el resultado de múltiples factores y no solo biológicos: Mejorar las condiciones de vida, luchar contra la distribución desigual del poder y medir la magnitud del problema, analizarlo y evaluar los efectos de las intervenciones es lo que propone la Comisión (21) y se ratifica como lineamiento mundial desde la misma 62 ${ }^{a}$ Asamblea Mundial de la Salud (22).

Lo que deja entrever esta situación, entre otras, es una crisis en la definición de lo que se entiende por calidad enla atención ala salud. Al respecto, conviene acudir a los aportes clásicos de Avedis Donabedian quien formuló que la calidad de la atención en salud se sustenta en siete pilares: Eficacia, Efectividad, Eficiencia, Optimización, Aceptabillidad, Legitimidad y Equidad (23). Sin embargo, a la hora de evaluar un programa o un servicio, Donabedian 
sugirió que debería diferenciarse claramente entre las dimensiones (Estructura, Proceso o Resultado) para definir el enfoque de la evaluación. Un enfoque estructural se centraría en aspectos como habilitación, acreditación, eficiencia; un enfoque de proceso se concentraría en revisión de casos o auditoría médica; en tanto que la evaluación desde un enfoque de resultados consiste en identificar los cambios que se producen en individuos o poblaciones, atribuibles al programa o servicio (24).

La Efectividad corresponde al enfoque de resultados y se define, en su versión más simple como "el grado en que una intervención produce resultados beneficiosos en el conjunto de la población diana" (25). Algunas revisiones han pretendido hacer esta evaluación en torno a programas para la prevención del suicidio (26-29). Inclusive, la Organización Mundial de la Salud realizó una juiciosa sistematización de programas basados en la evidencia (30). No obstante, estas revisiones no se concentran en las estrategias de base comunitaria (aunque tampoco las excluyen) y la definición de los indicadores es tan dispersa que va desde la medición de la tasa de suicidios hasta el aumento en conocimientos sobre la depresión, pasando por otras variables cognitivas como ideación suicida o actitudes hacia el suicidio. De hecho, la revisión realizada por York y sus colegas, que se concibe como el principal antecedente del presente estudio por centrarse en programas de base comunitaria, deja ver que los efectos más favorables se dieron en actitudes y conocimientos sobre el suicidio pero la reducción de los actos suicidas no fue igualmente prometedora (31). Revive la cuestión de si la efectividad de un programa se mide por sus resultados presuntamente intermediarios como lo sugirió Baranowski (19) o por aquello se espera afectar. Dicho de otro modo, los criterios para establecer efectividad se basan en resultados intermedios y no en resultados finales.

Al estudio de York y colegas también le recae el hecho de que, aunque fue publicado en 2013, la búsqueda se realizó para el lapso de 1970 a 2002, lo que supondría nuevos estudios en los últimos años, en respuesta a las múltiples recomendaciones como la de la Comisión sobre Determinantes Sociales de la Salud.
Por lo antedicho, se realiza la presente revisión sistemática que pretende evaluar la efectividad de los programas de base comunitaria para la reducción de las tasas de suicidio e intento de suicidio. Los resultados servirán de insumo para la toma de decisiones por parte de las autoridades sanitarias, teniendo en cuenta las intervenciones que demuestren mayor evidencia, lo mismo que para alimentar una línea de investigación necesaria en el Departamento del Huila.

\section{Metodología}

Criterios de elegibilidad: Para la revisión sistemática se llevó a cabo una búsqueda en las bases de datos y motores de búsqueda MEDLINE, Cochrane Library, Lilacs, Portal Regional da BVS, Psycarticles y EBSCO (Academic Search Complete). También se revisaron otras fuentes de literatura gris como tesis de grado y memorias de congresos.

Se optó por utilizar un mismo algoritmo para todas las bases con el fin de garantizar una mayor sistematicidad. El algoritmo utilizado fue "effectiveness community suicide prevention program" por reunir todos los términos que el equipo había discutido ampliamente sobre la base del objetivo principal. Elnúmero de registros se muestra en la figura 1 . No se discriminaron registros en razón a fecha de publicación, tipo de población ni idioma. Se aceptaron los siguientes tipos de estudio:

1. Ensayos clínicos

2. Ensayos comunitarios

3. Estudios ecológicos

4. Estudios cuasiexperimentales

5. Estudios observacionales de evaluación de programas

En cuanto a las intervenciones, la base comunitaria debía ser declarada explícitamente o descrita en el procedimiento, conforme a lo definido en la literatura.

Con referencia a los resultados reportados, se admitieron de acuerdo con la siguiente clasificación: 
1. Resultados Primarios: Los estudios elegidos debieron reportar

- Tasas de suicidio

- Tasas de Intento de Suicidio

2. Resultados secundarios: Se aceptaron estudios que reportaran variables intermediarias como

- Cogniciones relacionadas con suicidio, autolesiones $\mathrm{u}$ otras variables relacionadas.

- Actitudes frente al suicidio, autolesiones $\mathrm{u}$ otras variables relacionadas

- Mediciones relacionadas con la calidad de vida de las víctimas directas o indirectas de conductas suicidas

Fueron rechazados los siguientes tipos de documentos: 1- Artículos de reflexión o de desarrollo conceptual; 2- Artículos de evaluación de programas que se refieran a problemáticas distintas al suicidio; 3- Evaluación de programas de prevención del suicidio que no evidencien trabajo de base comunitaria; 4- Revisiones de programas o estrategias; 5- Protocolos de estudios.

Estrategia de búsqueda: La pesquisa inicial en las bases de datos fue realizada por FAS y una vez eliminados los registros duplicados mediante EndNote ${ }^{\circledR}$, FAShizo un primer barrido revisando títulos y resúmenes para aplicar los criterios de elegibilidad. Como paso siguiente, FAS y CFB descargaron la totalidad de los artículos para ser asignados de la siguiente manera: la mitad de los artículos le fue asignada a JCV y GJS; la otra mitad, a FAS y CFB. Cada uno de los miembros de la pareja leyó individualmente cada artículo y lo clasificaba como elegible o no elegible. Al término del ejercicio, los artículos clasificados coincidentemente pasaron a la siguiente fase; los artículos que generaron desacuerdo fueron objeto de discusión en el grupo hasta definir su destino.

Recolección de datos: Los artículos elegidos fueron sometidos a nueva lectura para extraer la siguiente información, que fue registrada en una planilla en Microsoft Excel:
1. Apellido del primer autor

2. País de ejecución

3. Año de publicación

4. Periodo de intervención

5. Población beneficiaria

6. Tipo de estudio

7. Descripción de la Intervención

8. Descripción de la comparación

9. Datos de Resultados Primarios

10. Datos de Resultados Secundarios

Se admitieron estudios que, aunque no abordaran los resultados primarios para este estudio, abordaban alguno o varios de los resultados secundarios.

Riesgo de sesgos de los estudios: La evaluación de la calidad de los estudios solo se aplicó a los estudios que abordaron los resultados primarios de la revisión. Para esto se utilizó la Herramienta de Evaluación de la Calidad para estudios Cuantitativos propuesta por el proyecto de Prácticas Efectivas en Salud Pública (disponible en http://www.ephpp.ca/ tools.html) y que aparece como una ayuda recomendada en las Guías de Revisiones Sistemáticas de Intervenciones de Promoción de la Salud y Salud Pública (32).

\section{Resultados}

Aplicando el algoritmo de búsqueda en las bases de datos relacionadas se recuperaron en total 136 registros, así:

Medline: 56

Cochrane Library: 6

Lilacs: 0

Portal Regional Da BVS: 64

Psycharticles: 7

EBSCO (Academic Search Complete): 3

Luego de eliminar duplicados, resultaron 84 registros.

Una vez surtido el primer barrido, permanecieron 53 registros, cuyos documentos fueron descargados para la lectura y definición de su permanencia. La lectura por binomios permitió la exclusión de 33 artículos más que 
no cumplieron con los criterios de elegibilidad, conformando una base para el presente estudio de 20 documentos. A continuación, se presenta el flujograma del proceso:

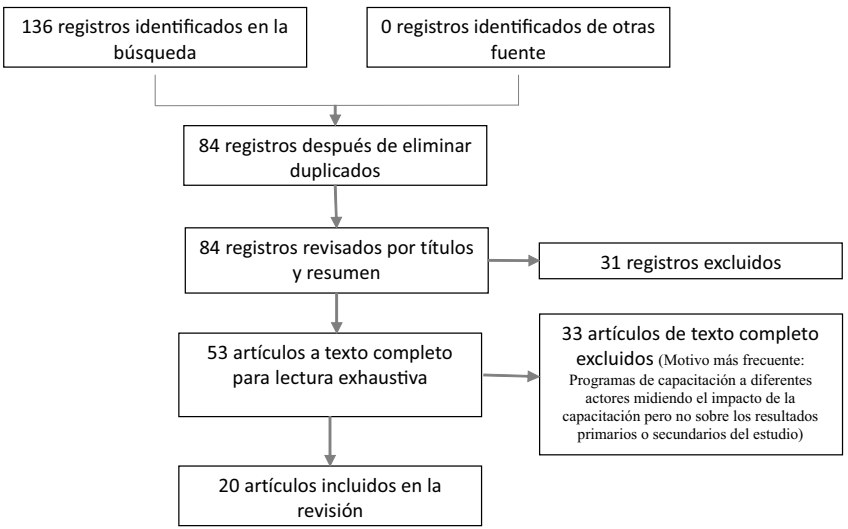

Figura 1. Diagrama de flujo

Los 33 artículos que fueron excluidos luego de la lectura exhaustiva, no cumplían con criterios de elegibilidad especialmente por no desarrollar estrategias consideradas como de base comunitaria, o por encontrar que los efectos se midieron en términos de variables que no correspondían a los resultados primarios o secundarios de interés para el presente estudio.

En la Tabla 1 se presenta el resumen descriptivo de los estudios revisados. De los 20 estudios revisados 11 (del número 10 al 20) establecieron como su criterio de efectividad en la prevención del suicidio la modificación de variables cognitivas (conocimiento), actitudinales o de otro tipo, que suelen ser relacionadas con los actos suicidas. Para la presente investigación estos son resultados secundarios. De otro lado, 9 estudios (del número 1 al 9) definieron su criterio de efectividad sobre la modificación de las estadísticas oficiales de actos suicidas, es decir, de las tasas de incidencia de suicidio e intento de suicidio. Un estudio evaluó los dos tipos de resultado (estudio número 6).
Tabla 1. Resumen descriptivo de los estudios revisados

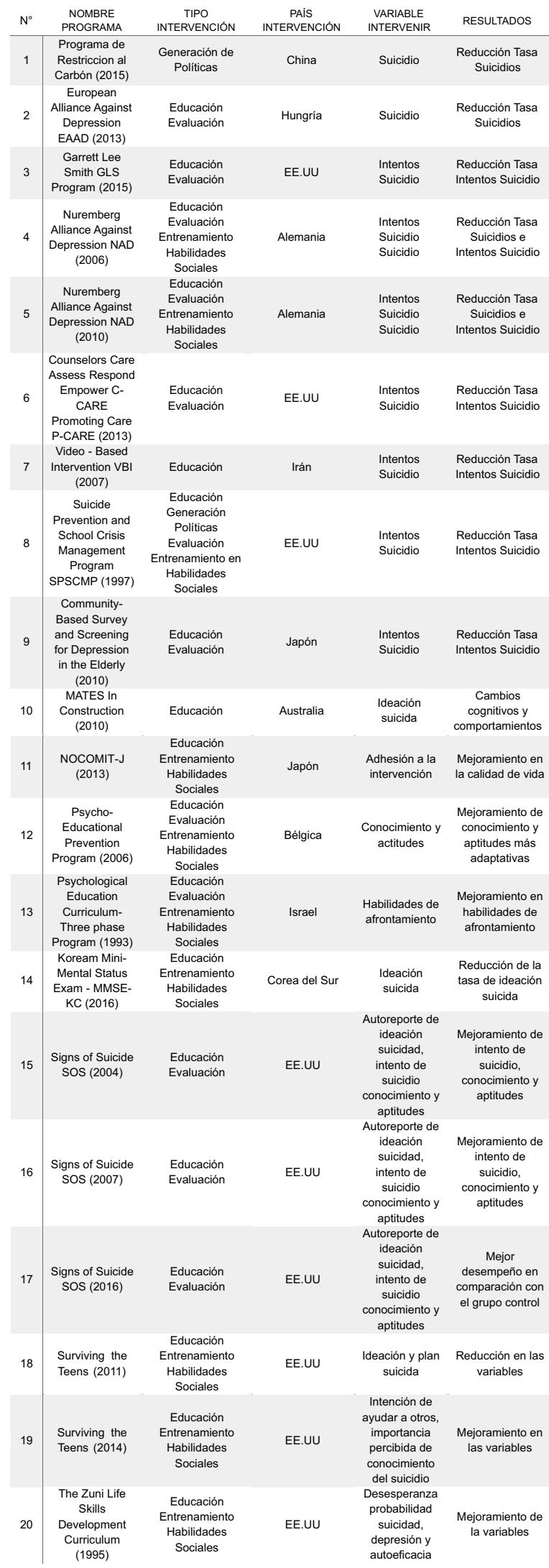


Varios de los estudios abordaron el mismo programa ya fuera en diferentes poblaciones o en diferentes momentos: El Programa European Alliance Against Deppression - EAAD (estudio 2) trabajó sobre la misma base del Nurenmerg Alliance against Deppression -NAD (estudios 4 y 5). Los estudios 15, 16 y 17 abordaron el Programa Signs of Suicide - SOS y los estudios 18 y 19 trabajaron sobre el programa Surviving the Teens. Dada esta circunstancia, los 20 estudios contemplan en total 15 programas cuyas intervenciones de base comunitaria se podrían resumir de la siguiente manera:

Tabla 2. Categorías de las Intervenciones

\begin{tabular}{l|l}
\multicolumn{1}{c}{ CATEGORÍA } & \multicolumn{1}{c}{ DESCRIPCIÓN } \\
\hline 1. EDUCACIÓN & $\begin{array}{l}\text { Programas que desarrollaron capacitaciones, formaciones, } \\
\text { talleres y paquetes dirigidos a la población con el objeti vo de } \\
\text { aumentar en conocimiento de los temas relacionados, } \\
\text { reconocimiento de signos de depresión. }\end{array}$ \\
2. GENERACIÓN DE POLÍTICA & $\begin{array}{l}\text { Programas que dentro de su estructura generaron políticas } \\
\text { generales que impartieron para orientar, restringir y } \\
\text { promocionar comportamientos de la población. } \\
\text { 3. EVALUACIÓN }\end{array}$ \\
$\begin{array}{l}\text { Programas que dentro de su estructura realizaron entrevistas, } \\
\text { tamizaje y aplicación de instrumentos, para la identificación de } \\
\text { patologías y evaluación de la intervención y de otros riesgos. } \\
\text { Programas que dentro de su estructura desarrollaron habilidades } \\
\text { en el manejo de afrontamiento de crisis, grupos de autoayuda, } \\
\text { de comunicación }\end{array}$ \\
HABILIDADES SOCIALES
\end{tabular}

Los diseños utilizados fueron en su mayoría cuasiexperimentos en los que se realizaron mediciones previas y posteriores a la intervención (comparaciones intragrupo) y también se utilizaron grupos de control (comparaciones intergrupos). Para todos los casos los resultados fueron favorables según las variables abordadas, aunque los efectos con mayor evidencia estadística se dieron entre los estudios que evaluaron variables intermedias.

En cuanto a la calidad de los estudios, en la Tabla 3 se puede evidenciar que los nueve estudios analizados mostraron que los riesgos de sesgo fueron altos, toda vez que ninguno desarrolló procedimientos rigurosos de selección (por tratarse estrategias de base poblacional), cegamiento, control de confusores, dificultad para el control de retiros y pérdidas y control de variables en la intervención.
Tabla 3. Evaluación de Riesgo de Sesgos

\begin{tabular}{c|cccccc}
$\begin{array}{c}\text { EStudio } \\
\mathbf{N}^{\circ}\end{array}$ & SELECCIÓN & DISEÑO & CONFUSORES & CEgAMIENTO & $\begin{array}{c}\text { RECOLECCIÓN } \\
\text { DE DATOS }\end{array}$ & ABANDONOS \\
\hline $\mathbf{1}$ & Débil & Débil & Débil & Débil & Débil & Débil \\
$\mathbf{2}$ & Débil & Débil & Débil & Débil & Débil & Débil \\
$\mathbf{3}$ & Débil & Débil & Débil & Débil & Débil & Débil \\
$\mathbf{4}$ & Débil & Débil & Débil & Débil & Débil & Débil \\
$\mathbf{5}$ & Débil & Débil & Débil & Débil & Débil & Débil \\
$\mathbf{6}$ & Débil & Débil & Débil & Débil & Débil & Débil \\
$\mathbf{7}$ & Débil & Débil & Débil & Débil & Débil & Débil \\
$\mathbf{8}$ & Débil & Débil & Débil & Débil & Débil & Débil \\
$\mathbf{9}$ & Débil & Débil & Débil & Débil & Débil & Débil
\end{tabular}

\section{Discusión}

La prevención del suicidio es, definitivamente un tema de prioridad indiscutible. La inversión hecha para reducir las tasas de los actos suicidas se justifica en tanto se trata de reducir la pérdida de vidas humanas. La base comunitaria se ha propuesto como una alternativa viable desde hace cuatro décadas para ser aplicada en los programas de prevención y promoción de la salud. No obstante, la rigurosidad en la aplicación de los programas no es una característica notoria, lo que termina por convertirse en la principal debilidad del enfoque. El déficit a la hora de definir marcos conceptuales, principalmente en programas multinivel que implementan diferentes tipos de estrategia, pero muy especialmente la tendencia a medir la efectividad de los programas para la prevención del suicidio en términos de variables que se considerarían intermedias, deja ver que aún falta un largo camino por recorrer. Pretender que un programa de prevención del suicidio se considere exitoso por lograr modificaciones en los conocimientos sobre el suicidio o la depresión o sobre las actitudes hacia la muerte es ciertamente inadecuado, pues proporciona una idea desviada de lo que se entendería como efectividad.ParaBaranowski(19)la explicaciónes clara: estamos utilizando teorías que se suponen poderosas predictoras de comportamientos que no necesariamente tienen la suficiente evidencia empírica. Para Elsa Moreno y sus colegas, siguiendo a Avedis Donabedian, la evaluación de los programas en Atención Primaria en Salud deberían suscribirse a uno de tres enfoques: de Estructura, de Proceso o de Resultados (24). En la línea que corresponde al presente estudio, la efectividad de los programas de prevención 
del suicidio debería medirse en términos de reducción de la tasa de suicidio. Otros efectos deben considerarse secundarios.

Otro aspecto que amerita consideración es la rigurosidad de los estudios que fue baja, con un alto riesgo de sesgo, desde la asignación de grupos, la aplicación de la intervención, hasta el manejo de los abandonos. Sin duda, la naturaleza de las intervenciones, así como la extensión de la población beneficiaria son factores que hacen difícil un control total de las variables. Los cuasiexperimentos son, por definición, ejercicios en los cuales dicho control es imposible. Es de esperar que no se realicen ensayos aleatorizados; pero se debe propender por elevar gradualmente la rigurosidad para alcanzar mejores niveles de evidencia, aunque implique al comienzo sacrificar extensión de los participantes. Un ejemplo de esto lo da el Programa MATES in Construction (estudio revisado número 10) que se concentró en una porción de la población, de manera que se hace más fácil la identificación y el control de las variables.

Como principales limitaciones de la presente investigación se plantean: la dificultad para la aplicación estandarizada de protocolos de evaluación de la calidad debido a la diversidad de estudios, y principalmente a criterio de los autores, el limitado tiempo para el desarrollo del ejercicio.

El estudio proporciona importantes ideas para la profundización en el tema y deja algunos elementos a considerar por parte de las autoridades sanitarias a la hora de tomar decisiones y guiar las estrategias en la prevención del suicidio. Las estrategias multimodales que incluyen educación, tamizaje, currículos y seguimiento, mostraron ser generosas en sus efectos, pese a las debilidades metodológicas en la demostración. Las estrategias de restricción no deberían ir solas, sino como parte de programas más robustos. De igual manera, queda claro que son los programas a los que se les realiza un seguimiento de años los que pueden demostrar de modo fehaciente su efectividad en términos de estadísticas.
Finalmente, los resultados ponen de manifiesto, al menos, tres condiciones de la realidad actual relacionada con la salud y la salud mental: 1) Faltan más intervenciones que involucren a la comunidad (en cualquiera de sus connotaciones); 2) Falta alinear el marco conceptual del diseño con los criterios para la evaluación del efecto de los programas; y 3) Falta identificar cuáles son los propósitos terminales de los programas, pues muchos se quedan en objetivos instrumentales.

\section{Agradecimientos}

Los autores manifiestan su sincero agradecimiento a los docentes Jorge Ramos y Norman Apraez, asesores metodológico y temático respectivamente.

\section{Contribuciones de los autores}

Todos los autores desarrollaron funciones similares a lo largo de la investigación $y$ preparación del manuscrito

\section{Declaración de interés}

Los autores manifiestan que no presentaron conflicto de intereses ni vinculación con ninguna institución u organismo mencionado en el estudio.

\section{Referencias bibliográficas}

1. World Health Organization. (2014). Preventing suicide: a global imperative. World Health Organization.

2. Winer, E. S., Drapeau, C. W., Veilleux, J. C., \& Nadorff, M. R. (2016). The association between anhedonia, suicidal ideation, and suicide attempts in a large student sample. Archives of suicide research, 20(2), 265-272.

3. Montoya-Gómez, B. (2015). Comportamiento del suicidio.

4. Porter, D., \& Bertolote, J. (2004). El suicidio, un problema de salud pública enorme y sin embargo prevenible, según la OMS. Recuperado el, 20, r61.

5. de la Salud, A. M. (2013). Plan de acción integral sobre salud mental 2013-2020. 
6. Castelpietra, G., Morsanutto, A., PascoloFabrici, E., \& Isacsson, G. (2008). Antidepressant use and suicide prevention: a prescription database study in the region Friuli Venezia Giulia, Italy. Acta Psychiatrica Scandinavica, 118(5), 382-388.

7. Cipriani, A., Hawton, K., Stockton, S., \& Geddes, J. R. (2013). Lithium in the prevention of suicide in mood disorders: updated systematic review and meta-analysis. Bmj, 346, f3646.

8. Gullestrup, J., Lequertier, B., \& Martin, G. (2011). MATES in construction: impact of a multimodal, community-based program for suicide prevention in the construction industry. International journal of environmental research and public health, 8(11), 4180-4196.

9. Ono, Y., Awata, S., Iida, H., Ishida, Y., Ishizuka, N., Iwasa, H., ... \& Nishi, N. (2008). A community intervention trial of multimodal suicide prevention program in Japan: a novel multimodal community intervention program to prevent suicide and suicide attempt in Japan, NOCOMIT-J. BMC public health, 8(1), 315.

10. Tarrier, N., Taylor, K., \& Gooding, P. (2008). Cognitive-behavioral interventions to reduce suicide behavior: a systematic review and metaanalysis. Behavior modification, 32(1), 77-108.

11. Rudd, M. D., Bryan, C. J., Wertenberger, E. G., Peterson, A. L., Young-McCaughan, S., Mintz, J., ... \& Wilkinson, E. (2015). Brief cognitivebehavioral therapy effects on post-treatment suicide attempts in a military sample: results of a randomized clinical trial with 2-year follow-up. American Journal of Psychiatry, 172(5), 441-449.

12. Rose, G. (2001). Sick individuals and sick populations. International journal of epidemiology, 30(3), 427-432.

13. IASP Executive Committee. (1999). IASP guidelines for suicide prevention. Crisis, 20(4), 155-163.

14. Delgado Garcia, G. (2008). Conferencia internacional sobre atención primaria de salud, Almá-Atá (1978). Cuadernos de Historia de la Salud Pública.

15. Hawe, P. (1994). Capturing the meaning of 'community' in community intervention evaluation: some contributions from community psychology. Health Promotion International, 9(3), 199-210.
16. Whitehead, T. (2002). Community based interventions, definitions and types. The cultural ecology of health and change (CEHC). Maryland: University of Maryland.

17. McLeroy, K. R., Norton, B. L., Kegler, M. C., Burdine, J. N., \& Sumaya, C. V. (2003). Community-based interventions.

18. Merzel, C., \& D'Afflitti, J. (2003). Reconsidering community-based health promotion: promise, performance, and potential. American journal of public health, 93(4), 557-574.

19. Baranowski, T., Lin, L. S., Wetter, D. W., Resnicow, K., \& Hearn, M. D. (1997). Theory as mediating variables: why aren't community interventions working as desired?. Annals of Epidemiology, 7(7), S89-S95.

20. Tognoni, G. (Ed.). (1997). Manual de epidemiología comunitaria. Cecomet.

21. Arellano, O. L., Escudero, J. C., \& Moreno, L. D. C. (2008). Los determinantes sociales de la salud: una perspectiva desde el Taller Latinoamericano de Determinantes Sociales sobre la Salud, ALAMES. Medicina Social, 3(4), 323-335.

22. de la Salud, A. M. (2009). Reducir las inequidades sanitarias actuando sobre los determinantes sociales de la salud.

23. Donabedian, A. (1990). The seven pillars of quality. Archives of Pathology and Laboratory Medicine.

24. Moreno, E. M., Crivelli, V., Kestelman, N., Malé, M., Passarelli, N., Schapira, E., \& Viñas, M. (1998). Calidad de la atención primaria: aporte metodológico para su evaluación. In Calidad de la atención primaria: aporte metodológico para su evaluación.

25. Nebot, M., López, M. J., Ariza, C., Villalbí, J. R., \& García-Altés, A. (2011). Evaluación de la efectividad en salud pública: fundamentos conceptuales y metodológicos. Gaceta sanitaria, $25,3-8$.

26. Mann, J. J., Apter, A., Bertolote, J., Beautrais, A., Currier, D., Haas, A., ... \& Mehlum, L. (2005). Suicide prevention strategies: a systematic review. Jama, 294(16), 2064-2074.

27. Zalsman, G., Hawton, K., Wasserman, D., van Heeringen, K., Arensman, E., Sarchiapone, M., ... \& Purebl, G. (2016). Suicide prevention strategies revisited: 10-year systematic review. The Lancet Psychiatry, 3(7), 646-659. 
28. O'Carroll, P. W., Potter, L. B., \& Mercy, J. A. (1994). Programs for the prevention of suicide among adolescents and young adults. Morbidity and Mortality Weekly Report: Recommendations and Reports, 1-7.

29. Cox, G. R., Owens, C., Robinson, J., Nicholas, A., Lockley, A., Williamson, M., .\& Pirkis, J. (2013). Interventions to reduce suicides at suicide hotspots: a systematic review. BMC public health, 13(1), 214.

30. World Health Organization. (2010). Towards evidence-based suicide prevention programmes.

31. York, J., Lamis, D. A., Friedman, L., Berman, A. L., Joiner, T. E., Mcintosh, J. L., ... \& Pearson, J. (2013). A systematic review process to evaluate suicide prevention programs: A sample case of community-based programs. Journal of community psychology, 41(1), 35-51.

32. Armstrong, R., Waters, E., \& Jackson, N. (2007). Systematic reviews of health promotion and public health interventions. Melbourne: University of Melbourne.

\section{Estudios revisados}

1. Chen, Y. Y., Chen, F., Chang, S. S., Wong, J., \& Yip, P. S. (2015). Assessing the efficacy of restricting access to barbecue charcoal for suicide prevention in Taiwan: a community-based intervention trial. PloS one, 10(8), e0133809.

2. Székely, A., Thege, B. K., Mergl, R., Birkás, E., Rózsa, S., Purebl, G., \& Hegerl, U. (2013). How to decrease suicide rates in both genders? An effectiveness study of a community-based intervention (EAAD). PloS one, 8(9), e75081.

3. Garraza, L. G., Walrath, C., Goldston, D. B., Reid, H., \& McKeon, R. (2015). Effect of the Garrett Lee Smith memorial suicide prevention program on suicide attempts among youths. JAMA psychiatry, 72(11), 1143-1149.

4. Hegerl, U., Althaus, D., Schmidtke, A., \& Niklewski, G. (2006). The alliance against depression: 2-year evaluation of a communitybased intervention to reduce suicidality. Psychological medicine, 36(9), 1225-1233.

5. Hegerl, U., Mergl, R., Havers, I., Schmidtke, A., Lehfeld, H., Niklewski, G., \& Althaus, D. (2010). Sustainable effects on suicidality were found for the Nuremberg alliance against depression. European archives of psychiatry and clinical neuroscience, 260(5), 401-406.

6. Hooven, C. (2013). Parents-CARE: A suicide prevention program for parents of at-risk youth. Journal of child and adolescent psychiatric nursing, 26(1), 85-95.

7. Ahmadi, A., \& Ytterstad, B. (2007). Prevention of self-immolation by communitybased intervention. Burns, 33(8), 1032-1040.

8. Zenere III, F. J., \& Lazarus, P. J. (1997). The decline of youth suicidal behavior in an urban, multicultural public school system following the introduction of a suicide prevention and intervention program. Suicide and LifeThreatening Behavior, 27(4), 387-403.

9. Oyama, H., Sakashita, T., Hojo, K., Ono, Y., Watanabe, N., Takizawa, T., \& Tanaka, E. (2010). A community-based survey and screening for depression in the elderly. Crisis.

10. Gullestrup, J., Lequertier, B., \& Martin, G. (2011). MATES in construction: impact of a multimodal, community-based program for suicide prevention in the construction industry. International journal of environmental research and public health, 8(11), 4180-4196.

11. Ono, Y., Sakai, A., Otsuka, K., Uda, H., Oyama, H., Ishizuka, N., ... \& Motohashi, Y. (2013). Effectiveness of a multimodal community intervention program to prevent suicide and suicide attempts: a quasi-experimental study. PLoS One, 8(10), e74902.

12. Portzky, G., \& Van Heeringen, K. (2006). Suicide prevention in adolescents: a controlled study of the effectiveness of a school-based psycho-educational program. Journal of Child Psychology and Psychiatry, 47(9), 910-918.

13. Klingman, A., \& Hochdorf, Z. (1993). Coping with distress and self harm: the impact of a primary prevention program among adolescents. Journal of adolescence, 16(2), 121.

14. Kim, J. P., \& Yang, J. (2017). Effectiveness of a community-based program for suicide prevention among elders with early-stage dementia: A controlled observational study. Geriatric nursing, 38(2), 97-105.

15. Aseltine Jr, R. H., \& DeMartino, R. (2004). An outcome evaluation of the SOS suicide prevention program. American Journal of Public Health, 94(3), 446-451. 
16. Aseltine, R. H., James, A., Schilling, E. A., \& Glanovsky, J. (2007). Evaluating the SOS suicide prevention program: a replication and extension. BMC Public Health, 7(1), 161.

17. Schilling, E. A., Aseltine, R. H., \& James, A. (2016). The SOS suicide prevention program: Further evidence of efficacy and effectiveness. Prevention science, 17(2), 157-166.

18. King, K. A., Strunk, C. M., \& Sorter, M. T. (2011). Preliminary effectiveness of surviving the Teens ${ }^{\circledR}$ Suicide Prevention and Depression Awareness Program on adolescents' suicidality and self-efficacy in performing help-seeking behaviors. Journal of school health, 81(9), 581-590.

19. Strunk, C. M., King, K. A., Vidourek, R. A., \& Sorter, M. T. (2014). Effectiveness of the Surviving the Teens ${ }^{\circledR}$ Suicide Prevention and Depression Awareness Program: An impact evaluation utilizing a comparison group. Health Education \& Behavior, 41(6), 605-613.

20. LaFromboise, T., \& Howard-Pitney, B. (1995). The Zuni life skills development curriculum: Description and evaluation of a suicide prevention program. Journal of Counseling Psychology, 42(4), 479.

\section{Apéndice}

Herramienta de Evaluación dela Calídad para estudios Cuantitativos

ULITY ASSESSMENT TOOL

FOR QUANTITATIVE STUDIES

\section{COMPONENT RATINGS}

\section{A) SELECTION BIAS}

(Q1) Are the individuals selected to participate in the study likely to be representative of the target population?

1 Very likely

2 Somewhat likely

3 Not likely

4 Can't tell

(Q2) What percentage of selected individuals agreed to participate?

$180-100 \%$ agreement

$260-79 \%$ agreement
3 less than $60 \%$ agreement

4 Not applicable

5 Can't tell

\begin{tabular}{|lccc|}
\hline RATE THIS SECTION & STRONG & MODERATE & WEAK \\
See dictionary & 1 & 2 & 3 \\
\hline
\end{tabular}

\section{B) STUDY DESIGN}

Indicate the study design

1 Randomized controlled trial

2 Controlled clinical trial

3 Cohort analytic (two group pre + post)

4 Case-control

5 Cohort (one group pre + post (before and after))

6 Interrupted time series

7 Other specify

8 Can't tell

Was the study described as randomized? If NO, go to Component $\mathrm{C}$.

No

Yes

If Yes, was the method of randomization described? (See dictionary)

No Yes

If Yes, was the method appropriate? (See dictionary)

No Yes

\begin{tabular}{|lccc|}
\hline RATE THIS SECTION & STRONG & MODERATE & WEAK \\
See dictionary & 1 & 2 & 3 \\
\hline
\end{tabular}

\section{C) CONFOUNDERS}

(Q1) Were there important differences between groups prior to the intervention?

1 Yes

2 No

3 Can't tell

The following are examples of confounders:

1 Race

2 Sex

3 Marital status/family

4 Age

5 SES (income or class)

6 Education

7 Health status

8 Pre-intervention score on outcome measure

(Q2) If yes, indicate the percentage of relevant confounders that were controlled (either in the design (e.g. stratification, matching) or analysis)? 
$180-100 \%$ (most)

$260-79 \%$ (some)

3 Less than $60 \%$ (few or none)

4 Can't Tell

\begin{tabular}{|lccc|}
\hline RATE THIS SECTION & STRONG & MODERATE & WEAK \\
See dictionary & 1 & 2 & 3 \\
\hline
\end{tabular}

\section{D) BLINDING}

(Q1) Was (were) the outcome assessor(s) aware of the intervention or exposure status of participants?

1 Yes

2 No

3 Can't tell

(Q2) Were the study participants aware of the research question?

1 Yes

$2 \mathrm{No}$

3 Can't tell

\begin{tabular}{|lccc|}
\hline RATE THIS SECTION & STRONG & MODERATE & WEAK \\
See dictionary & 1 & 2 & 3 \\
\hline
\end{tabular}

\section{E) DATA COLLECTION METHODS}

(Q1) Were data collection tools shown to be valid?

1 Yes

2 No

3 Can't tell

(Q2) Were data collection tools shown to be reliable?

1 Yes

$2 \mathrm{No}$

3 Can't tell

\begin{tabular}{|lccc|}
\hline RATE THIS SECTION & STRONG & MODERATE & WEAK \\
See dictionary & 1 & 2 & 3 \\
\hline
\end{tabular}

\section{F) WITHDRAWALS AND DROP-OUTS}

(Q1) Were withdrawals and drop-outs reported in terms of numbers and/or reasons per group?

1 Yes

$2 \mathrm{No}$

3 Can't tell

4 Not Applicable (i.e. one time surveys or interviews)
(Q2) Indicate the percentage of participants completing the study. (If the percentage differs by groups, record the lowest).

$180-100 \%$

$260-79 \%$

3 less than $60 \%$

4 Can't tell

5 Not Applicable (i.e. Retrospective case-control)

\begin{tabular}{|lcccc|}
\hline RATE THIS SECTION & STRONG & MODERATE & WEAK & \\
See dictionary & 1 & 2 & 3 & Not Applicable \\
\hline
\end{tabular}

\section{G) INTERVENTION INTEGRITY}

(Q1) What percentage of participants received the allocated intervention or exposure of interest?

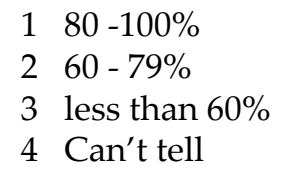

(Q2) Was the consistency of the intervention measured?

1 Yes

2 No

3 Can't tell

(Q3) Is it likely that subjects received an unintended intervention (contamination or cointervention) that may influence the results?
4 Yes
5 No
6 Can't tell

\section{H) ANALYSES}

(Q1) Indicate the unit of allocation (circle one) community organization/institution practice/office individual

(Q2) Indicate the unit of analysis (circle one) community organization/institution practice/ office individual

(Q3) Are the statistical methods appropriate for the study design?
1 Yes
2 No
3 Can't tell

(Q4) Is the analysis performed by intervention allocation status (i.e. intention to treat) rather than the actual intervention received?

$\begin{array}{ll}1 & \text { Yes } \\ 2 & \text { No } \\ 3 & \text { Can't tell }\end{array}$ 


\section{GLOBAL RATING}

\section{COMPONENT RATINGS}

Please transcribe the information from the gray boxes on pages 1-4 onto this page. See dictionary on how to rate this section.

\begin{tabular}{|c|c|c|c|c|c|}
\hline \multirow[t]{2}{*}{ A } & SELECTION BIAS & STRONG & MODERATE & WEAK & \\
\hline & & 1 & 2 & 3 & \\
\hline \multirow[t]{2}{*}{ B } & STUDY DESIGN & STRONG & MODERATE & WEAK & \\
\hline & & 1 & 2 & 3 & \\
\hline \multirow[t]{2}{*}{ c } & CONFOUNDERS & STRONG & MODERATE & WEAK & \\
\hline & & 1 & 2 & 3 & \\
\hline \multirow[t]{2}{*}{ D } & BLINDING & STRONG & MODERATE & WEAK & \\
\hline & & 1 & 2 & 3 & \\
\hline \multirow[t]{2}{*}{ E } & $\begin{array}{l}\text { DATA COLLECTION } \\
\text { METHOD }\end{array}$ & STRONG & MODERATE & WEAK & \\
\hline & & 1 & 2 & 3 & \\
\hline \multirow[t]{2}{*}{$\mathbf{F}$} & $\begin{array}{l}\text { WITHDRAWALS AND } \\
\text { DROPOUTS }\end{array}$ & STRONG & MODERATE & WEAK & \\
\hline & & 1 & 2 & 3 & Not Applicable \\
\hline
\end{tabular}

GLOBAL RATING FOR THIS PAPER (circle one):

$\begin{array}{lll}1 & \text { STRONG } & \text { (no WEAK ratings) } \\ 2 & \text { MODERATE } & \text { (one WEAK rating) } \\ 3 & \text { WEAK } & \text { (two or more WEAK ratings) }\end{array}$

With both reviewers discussing the ratings:

Is there a discrepancy between the two reviewers with respect to the component (A-F) ratings?

No Yes

If yes, indicate the reason for the discrepancy

1 Oversight

2 Differences in interpretation of criteria

3 Differences in interpretation of study

Final decision of both reviewers (circle one):

$\begin{array}{ll}1 & \text { STRONG } \\ 2 & \text { MODERATE } \\ 3 & \text { WEAK }\end{array}$




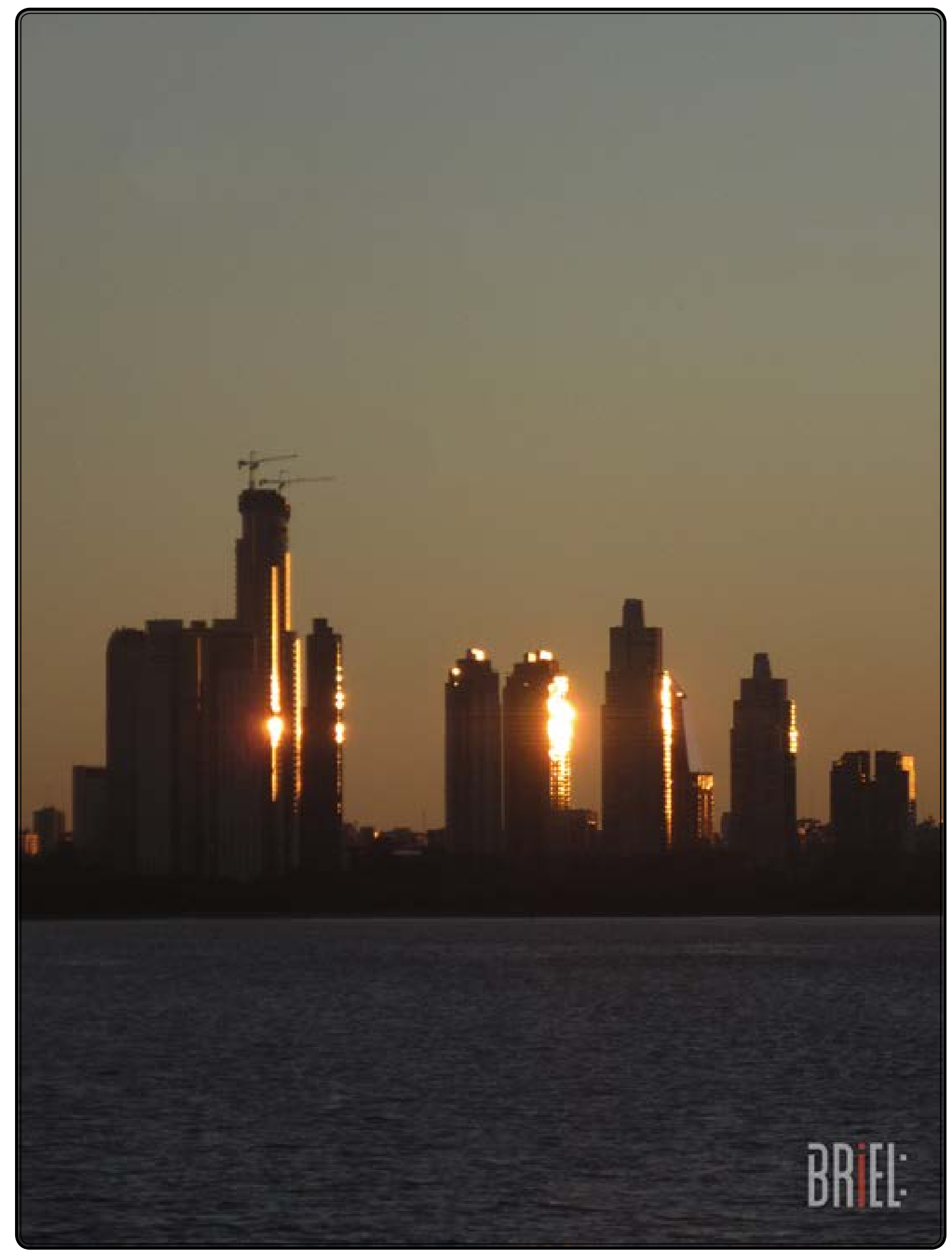

Un destello en la oscuridad somos. 